\title{
Dopaminergic pathway and primary visual cortex are involved in the freezing of gait in Parkinson's disease: a PET-CT study
}

This article was published in the following Dove Press journal: Neuropsychiatric Disease and Treatment

\section{Yongtao Zhou' \\ Junwu Zhao ${ }^{1,2}$ \\ Yaqin $\mathrm{Hou}^{3}$ \\ Yusheng $\mathrm{Su}^{3}$ \\ Piu Chan' \\ Yuping Wang'}

'The Department of Neurology, Xuanwu Hospital of Capital Medical University, Beijing, People's Republic of China; ${ }^{2}$ The Nuclear Medicine Department, Xuanwu Hospital of Capital Medical University, Beijing, People's Republic of China; ${ }^{3}$ The Department of Neurology, Weihai Municipal Hospital, Shandong, People's Republic of China
Correspondence: Yusheng Su

The Nuclear Medicine Department, Xuanwu Hospital of Capital Medical University, No.45 Changchun Street, Xicheng District, Beijing 100053, People's Republic of China

Tel +86 1065124875

Email xwyushengsu2018@I26.com

Piu Chan

The Department of Neurology, Xuanwu Hospital of Capital Medical University,

No.45 Changchun Street, Xicheng

District, Beijing 100053, People's Republic of China

Tel +861083198677

Email pbchan90@gmail.com
Background: Freezing of gait (FOG) could be partly alleviated by dopaminergic drugs but the mechanism still needs to be elucidated. The purpose of this study is to explore the mechanisms of FOG by vesicular monoamine transporter VMAT2 distribution with the ${ }^{18} \mathrm{~F}$-AV133 tracer and 18-fludeoxyglucose positron emission tomography-computed tomography $\left({ }^{18} \mathrm{~F}-\mathrm{FDG}\right.$ PET-CT).

Methods: Clinical material and PET-CT data were collected from 20 patients with FOG and 147 patients without FOG from November 1, 2017 to October 31, 2018. Brain scans of all participants were acquired over an approximately 20-min period, $120 \mathrm{~min}$ after injection of approximately $250 \mathrm{MBq}{ }^{18} \mathrm{~F}-\mathrm{AV} 133$. The mean uptake ratios of different regions were identified by NeuroQ software of ${ }^{18}$ F-FDG PET-CT. Data analysis included variance, chisquare analysis, covariance analysis, and logistic regression.

Results: Our data showed that patients with FOG were provided with greater doses of dopaminergic drugs $(p<0.05)$. The frequency of FOG was $11.98 \%$ and increased as Parkinson's disease progressed. FOG was more common in the elderly and strongly associated with the duration. Cognitive impairments were obvious, assessed by Mini-Mental State Examination and Montreal Cognitive Assessment $(p<0.05)$. The VMAT2 distribution with ${ }^{18} \mathrm{~F}$-AV133 was decreased significantly in the caudate nucleus and lentiform nucleus while the metabolism of these areas was elevated, determined by ${ }^{18}$ F-FDG PET-CT $(p<0.05)$. The metabolism of the primary visual cortex decreased obviously in patients with FOG compared with those without FOG $(p<0.05)$.

Conclusion: FOG mainly occurred in the advanced stage, and was strongly associated with the duration and larger dose of dopaminergic drugs. The dopamine level of the nigrostriatal system decreased significantly and the uptake ratios of the primary visual cortex dropped obviously in the FOG group compared with the non-FOG group. Our study suggests that both the dopaminergic pathway and the primary visual cortex are involved in the pathogenesis of FOG.

Keywords: freezing of gait, Parkinson's disease, clinical characters, VMAT2, positron emission tomography-computed tomography

\section{Introduction}

Freezing of gait (FOG) mainly occurs in the advanced stage of Parkinson's disease (PD) which leads to higher fall risk and poorer prognosis. It is mainly manifested by a sudden transient gait disorder during walking, most commonly when starting to walk and turning. ${ }^{1}$ FOG is a phenomenon that occurs most often during the "off" state, but it may also happen during the "on" state. The relationship between FOG and 


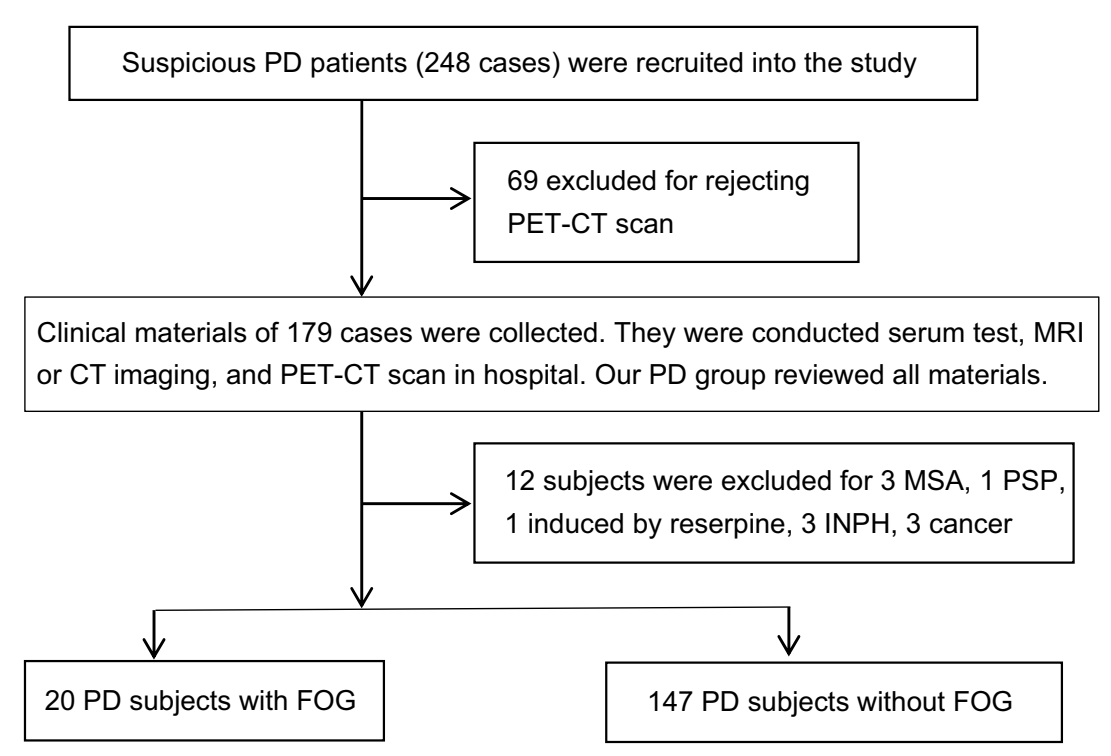

Figure I Flow chart of all subjects through the study.

Abbreviations: PD, Parkinson's disease; PET-CT, positron emission tomography-computed tomography; MRI, magnetic resonance imaging; MSA, multisystem atrophy; PSP, progressive nuclear paralysis; INPH, idiopathic normal pressure hydrocephalus; FOG, freezing of gait.

dopaminergic medication is complex. Most FOG could be relieved by levodopa dose adjustments, particularly those with FOG during the "off" state. ${ }^{2,3}$ But "on" FOG may be induced by dopaminergic drugs. ${ }^{4}$ The mechanism of freezing gait is not completely clear. Lesions of the cortex, brainstem, and basal ganglia were reported to be involved in the pathogenesis of FOG. The study of basal ganglia by molecular imaging has attracted more attention. ${ }^{5-7}$ Many studies have been carried out using dopamine transporter imaging worldwide and the results are inconsistent. ${ }^{8-11}{ }^{18} \mathrm{~F}-\mathrm{AV} 133$ is a tracer targeting for the vesicular monoamine transporter (VMAT2), which has little metabolism in the body and has better stability. ${ }^{12,13}$ There are few reports of basal ganglia lesions in this way. The aim of this study was to explore the mechanisms of FOG and provide exact evidence for the management of FOG.

\section{Methods}

\section{Standard protocol approvals and patient consents}

The present study was approved by the Ethics Committee of Xuanwu Hospital, Capital Medical University, Beijing, People's Republic of China. The methods were carried out in accordance with the approved guidelines. Written informed consent was obtained from all subjects. This study complied with the guidelines of Capital Medical University, which abided by the Helsinki Declaration on ethical principles for medical research.

\section{Study population}

All cases were recruited consecutively from inpatients of the neurological department at Xuanwu Hospital, Capital Medical University of China. PD was established as a probable disease by the movement disorder specialists according to the criteria of the UK Brain Bank. Patients with secondary parkinsonism or other forms of atypical parkinsonism were excluded. The first cardinal feature and the age of appearance were reviewed rigorously and supervised by neurologists and specialists (see figure 1).

\section{The definition of FOG}

The status of FOG was assessed on admission by their doctors in charge and experienced neurologists from clinical observation and inquiry with the patient himself/herself or caregivers. We also determined FOG by patients' responses to the question: "Do you feel that your feet get glued to the floor while walking, making a turn, or when trying to initiate walking?" Patients who answered "yes" were identified as freezers.

\section{Clinical assessments}

We selected the time of discharge to assess the score for the Hoehn-Yahr stage ${ }^{14}$ and the unified Parkinson's disease rating scale (UPDRS), ${ }^{15}$ which were used to evaluate the disease severity of PD patients. ${ }^{15}$ The Mini-Mental State Examination (MMSE) and Montreal Cognitive Assessment (MoCA) were administered to all of the 
enrolled subjects in order to assess the presence of cognitive impairment. ${ }^{16,17}$ To evaluate the role of pharmacotherapy, the levodopa equivalent dose was calculated for all of the PD patients. ${ }^{18}$

\section{${ }^{18} \mathrm{~F}-\mathrm{AVI} 33$ PET and CT image processing} The $\left[{ }^{18} \mathrm{~F}\right]$-DTBZ radiotracer $\left({ }^{18} \mathrm{~F}\right.$-AV 133) was prepared from aqueous $\left[{ }^{18} \mathrm{~F}\right]$-fluoride. Brain scans were acquired over an approximately 20-min period, $120 \mathrm{~min}$ after injection of approximately $250 \mathrm{MBq}$ of ${ }^{18} \mathrm{~F}$-AV133. Images were reconstructed using iterative reconstruction. Florbenazine images were spatially normalized to a standard atlas using an ${ }^{18}$ F-AV133 PET template. Atlas-based volumes of interest were applied for target areas (caudate, anterior and posterior putamen, and total striatum). The ratio of tracer activity in the target volumes of interest relative to the occipital cortex as a reference region was calculated to create the standard uptake value ratio. ${ }^{31-33}$

Three experienced radiologists read the image together, observed it visually, and synthesized the three axial images to judge the distribution of radioactivity in each part. The metabolic status of perivascular cortex or subcortical nucleus was assessed by 18-fludeoxyglucose positron emission tomography-computed tomography $\left({ }^{18}\right.$ F-FDG PET-CT) and compared with the contralateral cerebral hemisphere. NeuroQ software (Philip Company) was used to process the ${ }^{18}$ F-FDG PET-CT brain imaging. After rigid deformation, the whole brain was divided into 24 regions. By comparison with the reference area, the metabolism of each region was calculated. The cerebellum was defined as the reference region because the Lewy body of cerebellar pathology has not been demonstrated unequivocally in different stages of PD. The most decreased ${ }^{18} \mathrm{~F}$-FDG uptake regions of PET images were identified by NeuroQ software. The brain areas were automatically outlined by Scenium software and the mean standard uptake value was calculated between the two groups.

\section{Statistical analysis}

Analyses were performed with Statistical Package for the Social Sciences (SPSS) statistics (version 20.0; IBM Corporation, Armonk, NY, USA). The sex difference was explored between the FOG group and the non-FOG group by chi-square analysis and logistic regression. The data of age, age of onset, duration, MMSE, MoCA, equivalent and current levodopa dose, VMAT2, and brain region uptake ratio were analyzed by variance and covariance between the FOG group and the non-FOG group. The correlation of
FOG with clinical features, dopamine level, and uptake ratio of brain regions was conducted by bicorrelation and partial correlation. The significant difference was $p<0.05$.

\section{Results}

\section{Risk factors and predictors of freezing gait}

A total of 167 cases with PD were recruited into the study which included 20 cases (11.98\%) with FOG and 147 cases $(88.02 \%)$ without FOG. The mean age was 66.86 \pm 2.57 years in PD patients with FOG while it was 56.46 \pm 0.94 years in those without FOG. PD patients with FOG were older than those without FOG after adjusting for sex by covariate analysis $(p<0.05)$. The age of onset was not significantly different between the FOG group and the non-FOG group after adjusting for sex by covariate analysis (56.17 \pm 3.12 years vs $52.48 \pm 1.15$ years, $p>0.05)$. The duration of PD was significantly longer in the FOG group than that in the non-FOG group $(11.93 \pm 1.23$ years vs 3.69 \pm 0.44 years, $p<0.05$ ). The ratios of FOG were $4.17 \%$, $11.54 \%$, and $47.06 \%$, respectively, at no more than 2 years, 2-10 years, and more than 10 years of the disease. A sex difference was not observed whether PD patients had FOG or not (11.22 years vs $13.02 \%, p>0.05)$ after adjusting for age by logistic regression (11.22 years vs $13.02 \%, p>0.05)$.

The scores of Hoehn-Yahr stage and UPDRS-III were not significantly higher in patients with FOG than in those without FOG by variance and covariance analysis $(2.20$ \pm 0.15 and $41.04 \pm 4.29$ vs $2.00 \pm 0.06$ and $37.54 \pm 1.36$, $p>0.05)$. The dose of levodopa was higher in the FOG group compared with that in the non-FOG group after adjusting for age and sex by covariance (738.56 $\pm 80.37 \mathrm{mg}$ vs $530.90 \pm 28.68 \mathrm{mg}, p<0.05$ ).

The mean scores of MMSE and MoCA were 20.29 \pm 1.15 and $20.41 \pm 2.69$, respectively, in patients with FOG, and higher than those in PD patients without FOG $(p<0.05)$. After adjusting for age and sex by covariance analysis, there were still significant differences of MMSE and MoCA between the two groups $(23.49 \pm 1.04$ and $21.63 \pm 1.03$ vs $26.96 \pm 0.35$ and $24.43 \pm 0.35, p<0.05$ ) (see Table 1 ).

\section{The difference of dopamine level shown by VMAT2 with ${ }^{18} \mathrm{~F}-\mathrm{AV} 133$ between the two groups}

The mean uptake ratio of VMAT2 with ${ }^{18} \mathrm{~F}$-AV133 in the putamen was significantly lower in the FOG group than in the non-FOG group $(6.51 \pm 0.29$ vs $7.46 \pm 0.26, p<0.05)$. 
Table I Analysis of clinical characteristics in PD patients with FOG

\begin{tabular}{|c|c|c|c|c|c|c|}
\hline \multirow[t]{2}{*}{ Characteristic } & \multicolumn{3}{|c|}{ Before adjustment } & \multicolumn{3}{|c|}{ After adjustment } \\
\hline & FOG group & Non-FOG group & $p$ & FOG group & Non-FOG group & $p$ \\
\hline Age (years), mean $\pm S D$ & $66.90 \pm 1.51$ & $56.33 \pm 0.98$ & 0.00 & $66.86 \pm 2.57$ & $56.46 \pm 0.94$ & 0.00 \\
\hline Age of onset (years), mean $\pm S D$ & $56.25 \pm 3.20$ & $52.47 \pm 1.15$ & 0.26 & $56.17 \pm 3.12$ & $52.48 \pm 1.15$ & 0.27 \\
\hline $\begin{array}{l}\text { Duration } \\
\qquad 2 \text { years, } \mathrm{n}(\%) \\
2-10 \text { years, } \mathrm{n}(\%) \\
\geq 10 \text { years, } \mathrm{n}(\%)\end{array}$ & $\begin{array}{l}10.65 \pm 2.25 \\
3(4.17) \\
9(11.54) \\
8(47.06)\end{array}$ & $\begin{array}{l}3.86 \pm 0.38 \\
69(95.83) \\
69(88.46) \\
9(52.94)\end{array}$ & 0.00 & $11.93 \pm 1.23$ & $3.69 \pm 0.44$ & 0.00 \\
\hline $\begin{array}{l}\text { Sex } \\
\qquad \text { Male, n (\%) } \\
\text { Female, n (\%)) }\end{array}$ & $\begin{array}{l}\text { II (II.22) } \\
9 \text { (I3.04) }\end{array}$ & $\begin{array}{l}87(88.78) \\
60(86.96)\end{array}$ & 0.72 & $\begin{array}{l}\text { II (II.22) } \\
9(13.04)\end{array}$ & $\begin{array}{l}87(88.78) \\
60(86.96)\end{array}$ & 0.88 \\
\hline Hoehn-Yahr stage & $2.25 \pm 0.12$ & $1.99 \pm 0.06$ & 0.10 & $2.20 \pm 0.15$ & $2.00 \pm 0.06$ & 0.21 \\
\hline UPDRS & $59.25 \pm 1.96$ & $68.30 \pm 4.95$ & 0.53 & $47.53 \pm 12.36$ & $69.72 \pm 4.21$ & 0.09 \\
\hline UPDRS-III & $43.36 \pm 4.02$ & $37.30 \pm 1.39$ & 0.18 & $41.04 \pm 4.29$ & $37.54 \pm 1.36$ & 0.44 \\
\hline MMSE & $22.4 I \pm I .49$ & $27.09 \pm 0.34$ & 0.00 & $23.49 \pm 1.04$ & $26.96 \pm 0.35$ & 0.00 \\
\hline $\mathrm{MoCA}$ & $20.29 \pm 1.15$ & $24.60 \pm 0.37$ & 0.00 & $21.63 \pm 1.03$ & $24.43 \pm 0.35$ & 0.01 \\
\hline Equivalent levodopa dose (mg) & $704.08 \pm 51.34$ & $535.59 \pm 29.61$ & 0.04 & $738.56 \pm 80.37$ & $530.90 \pm 28.68$ & 0.02 \\
\hline
\end{tabular}

Notes: Single-factor analysis by variance and chi-square analysis. Adjusting confounding factors by covariate and logistic analysis. ${ }^{*} p<0.05$.

Abbreviations: PD, Parkinson's disease; FOG, freezing of gait; UPDRS, unified Parkinson's disease rating scale; MMSE, Mini-Mental State Examination; MoCA, Montreal Cognitive Assessment.

After adjusting for age and sex, the uptake ratio of VMAT2 was still lower in patients in the FOG group than in the non-FOG group $(6.26 \pm 0.51$ vs $7.52 \pm 0.18$, $p<0.05$ ). We further stratified the putamen into the anterior and dorsal putamen and found that the uptake ratio of VMAT2 decreased more obviously in the dorsal putamen in patients with FOG than in those without FOG after adjusting for age and sex by covariance $(2.77 \pm 0.25$ vs $3.32 \pm 0.09, p<0.05)$. Single-factor analysis found that the uptake ratio of VMAT2 in the caudate nucleus was significantly lower in the FOG group compared with that in the non-FOG group $(4.43 \pm 0.25$ vs $5.36 \pm 0.14, p<0.05)$. But there was no significant difference of VMAT2 distribution in the caudate nucleus between the two groups after adjusting for age and sex by covariance analysis $(p>0.05)$ (see Table 2 and Figures 2, 3, 4, 5).

Figure 2 shows that the dopamine level of the nigrostriatal system decreased significantly in the FOG group compared with the non-FOG group.

Figure 3 shows that the dopamine level of the nigrostriatal system declined obviously in a patient with FOG at Hoehn-Yahr stage 3 compared with a normal person and a patient with non-FOG.
Table 2 Uptake ratio of VMAT2 distribution by ${ }^{18} \mathrm{~F}-\mathrm{FDG}$ PET-CT in PD patients with FOG

\begin{tabular}{|l|l|l|l|l|l|l|}
\hline \multirow{2}{*}{$\begin{array}{l}\text { Brain } \\
\text { region }\end{array}$} & \multicolumn{2}{|l|}{ Before adjustment } & \multicolumn{2}{|c|}{ After adjustment } \\
\cline { 2 - 7 } & $\begin{array}{l}\text { FOG } \\
\text { group }\end{array}$ & $\begin{array}{l}\text { Non- } \\
\text { FOG } \\
\text { group }\end{array}$ & $\boldsymbol{P}$ & $\begin{array}{l}\text { FOG } \\
\text { group }\end{array}$ & $\begin{array}{l}\text { Non- } \\
\text { FOG } \\
\text { group }\end{array}$ & $\boldsymbol{P}$ \\
\hline Caudate & 4.43 & 5.36 & 0.02 & 4.74 & 5.32 & 0.15 \\
nucleus & \pm 0.25 & \pm 0.14 & & \pm 0.36 & \pm 0.14 & \\
Putamen & 6.51 & 7.46 & 0.07 & 6.26 & 7.52 & 0.02 \\
& \pm 0.29 & \pm 0.26 & & \pm 0.51 & \pm 0.18 & \\
Anterior & 3.59 & 4.18 & 0.05 & 3.50 & 4.20 & 0.02 \\
putamen & \pm 0.17 & \pm 0.11 & & \pm 0.29 & \pm 0.10 & \\
Dorsal & 2.92 & 3.30 & 0.15 & 2.77 & 3.32 & 0.04 \\
putamen & \pm 0.15 & \pm 0.10 & & \pm 0.25 & \pm 0.09 & \\
\hline
\end{tabular}

Notes: Single-factor analysis by variance and adjusting confounding factors by covariate analysis. ${ }^{*} p<0.05$.

Abbreviations: ${ }^{18} \mathrm{~F}$-FDG PET-CT, 18-fludeoxyglucose positron emission tomography-computed tomography; PD, Parkinson's disease; FOG, freezing of gait.

Figure 4 shows that the dopamine level of the nigrostriatal system declined obviously in a patient without FOG at Hoehn-Yahr stage 3 compared with a normal person and was higher than that in a patient with FOG.

Figure 5 shows that the dopamine level of the nigrostriatal system was normal. 


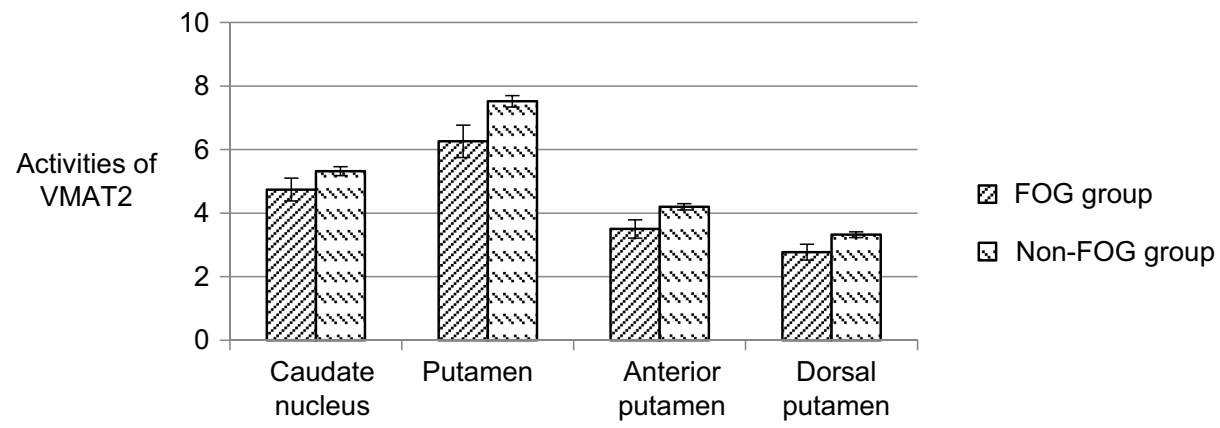

Figure 2 Activities of VMAT2 between the two groups. Abbreviation: FOG, freezing of gait.

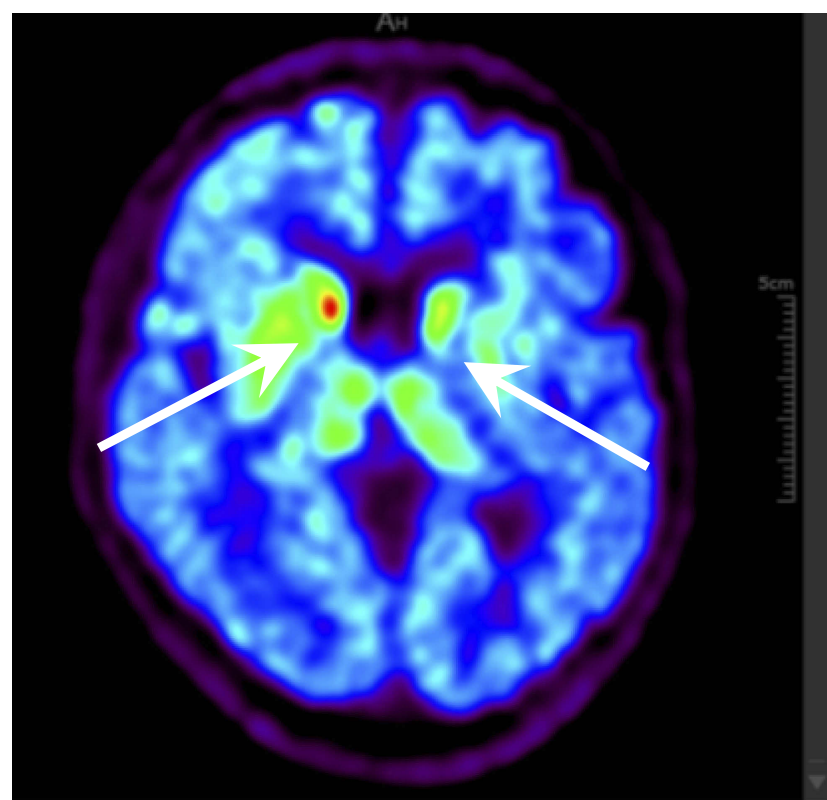

Figure 3 PET-CT image of VMAT2 distribution in a Hoehn-Yahr stage 3 patient with freezing of gait. Two white arrows indicate severe dopamine decline of bilateral putamens in cases.

Abbreviation: PET-CT, positron emission tomography-computed tomography.

\section{The association between FOG and activities of brain regions by ${ }^{18} \mathrm{~F}-\mathrm{FDG}$ PET-CT in PD}

The whole brain was divided into 24 regions by NeuroQ software. Our data found that the mean uptake ratios of the caudate nucleus and lentiform nucleus were higher in patients with FOG than those without FOG by variance analysis $(p<0.05)$. After adjusting for age and sex by covariance, the mean uptake ratios of the caudate nucleus and lentiform nucleus were still higher in the FOG group compared with those without FOG $(p<0.05)$. The mean uptake ratio of the left primary visual cortex was significantly lower in the FOG group than that in the non-FOG group after adjusting for age and sex by covariance analysis $(p<0.05)$. There were no differences of uptake ratios detected by

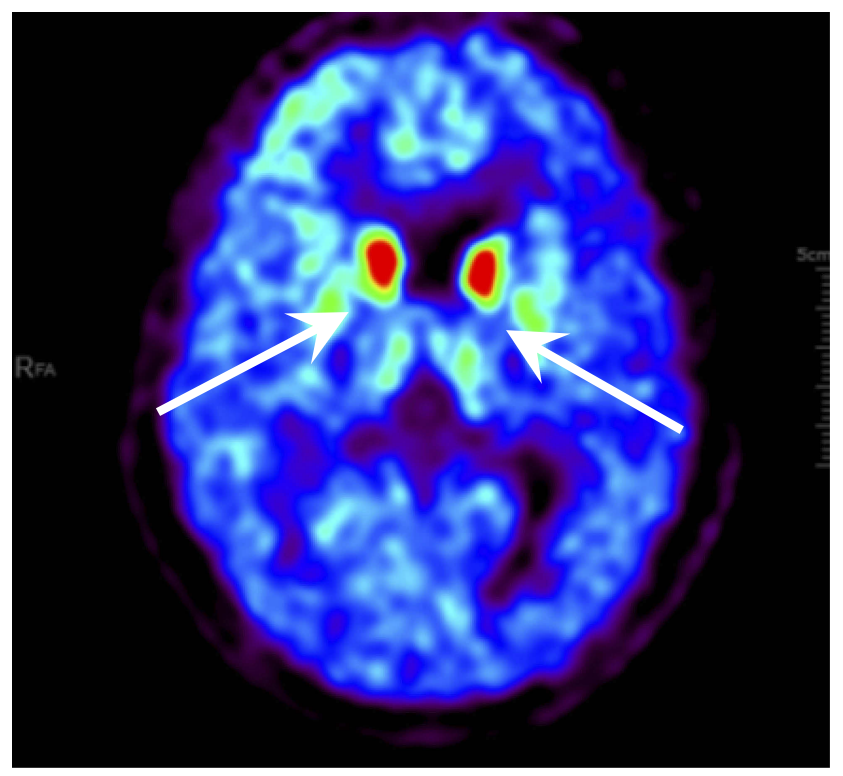

Figure 4 PET-CT image of VMAT2 distribution in a Hoehn-Yahr stage 3 patient without freezing of gait. Two white arrows show the dopamine decline of bilateral putamen.

Abbreviation: PET-CT, positron emission tomography-computed tomography.

${ }^{18}$ F-FDG PET-CT in other brain areas between the FOG group and the non-FOG group by variance and covariance analysis $(p>0.05$ ) (see Table 3 and Figures $6,7,8,9$ ).

Figure 6 shows that activities of the nigrostriatal system increased significantly in the FOG group compared with the non-FOG group while the activity of the primary visual cortex decreased obviously in the FOG group.

Figure 7 shows that the activity of the primary visual cortex decreased obviously in a patient with FOG compared with a normal person (Figure 9) and a non-FOG PD patient (Figure 8).

\section{Discussion}

FOG is a common disabling symptom mainly at the advanced stage of disease and the prevalence has varied 


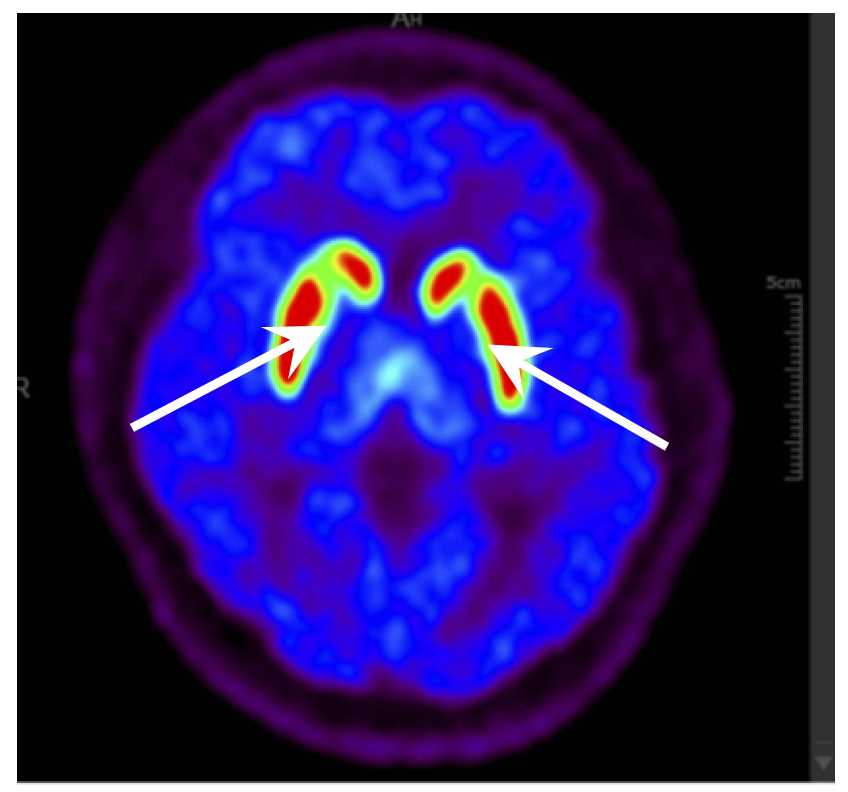

Figure 5 PET-CT image of VMAT2 distribution in a normal person. Two white arrows show the normal dopamine level of bilateral putamen.

Abbreviation: PET-CT, positron emission tomography-computed tomography.

from $7 \%$ in $\mathrm{PD}$ patients with a recent diagnosis to $63 \% .^{19,20,21}$ FOG prevalence reflects a mixed population of patients with early and advanced disease, most of whom could be improved by dopaminergic drugs. The ratio of FOG was $11.98 \%$ in our study and mainly occurred in the elderly. This frequency was lower than that of previous studies in China, and may be due to the hospital studied and patients selected. Male predominance was not observed in PD patients with FOG, in which the ratio was $11.22 \%$.

Our data found that the ratio of FOG increased as the disease progressed and was strongly related to the duration. The ratio of FOG ranged from $4.17 \%$ at 2 years of the disease to $47.06 \%$ after 10 years. The dose of equivalent levodopa was also significantly higher in patients with FOG at discharge than in persons without FOG. This result suggested that patients with FOG needed more dopaminergic drugs in order to improve motor symptoms and reach a nearly normal life compared with those without FOG. This phenomenon was also observed in other studies in which patients with FOG needed more dopaminergic drugs and were mainly in the advanced stage of PD. ${ }^{21,22}$ Our data did not find that FOG was associated with the motor severity according to the scores of Hoehn-Yahr

Table 3 Uptake ratios of different brain regions by ${ }^{18}$ F-FDG PET-CT in PD patients with FOG

\begin{tabular}{|c|c|c|c|c|c|c|}
\hline \multirow[t]{2}{*}{ Brain region } & \multicolumn{3}{|c|}{ Before adjustment } & \multicolumn{3}{|c|}{ After adjustment } \\
\hline & FOG group & Non-FOG group & $p$ & FOG group & Non-FOG group & $p$ \\
\hline Midbrain & $0.85 \pm 0.03$ & $0.84 \pm 0.01$ & 0.49 & $0.87 \pm 0.02$ & $0.84 \pm 0.01$ & 0.14 \\
\hline Caudate nucleus & $1.10 \pm 0.03$ & $1.07 \pm 0.0 \mathrm{I}$ & 0.16 & $1.12 \pm 0.02 *$ & $1.06 \pm 0.01$ & 0.03 \\
\hline Lentiform nucleus & $|.4| \pm 0.04^{*}$ & $1.32 \pm 0.01$ & 0.01 & $1.40 \pm 0.03 *$ & $\mathrm{I} .33 \pm 0.0 \mathrm{I}$ & 0.045 \\
\hline Thalamus & $1.2 \mathrm{I} \pm 0.04$ & $1.18 \pm 0.01$ & 0.21 & $1.21 \pm 0.03$ & $1.18 \pm 0.01$ & 0.18 \\
\hline Medial frontal cortex & $1.15 \pm 0.03$ & $1.13 \pm 0.01$ & 0.64 & $1.14 \pm 0.03$ & $1.13 \pm 0.01$ & 0.85 \\
\hline Superior frontal cortex & $1.14 \pm 0.04$ & $1.10 \pm 0.01$ & 0.21 & $1.14 \pm 0.03$ & $1.10 \pm 0.01$ & 0.27 \\
\hline Middle frontal cortex & $1.24 \pm 0.04$ & $1.21 \pm 0.01$ & 0.30 & $1.24 \pm 0.03$ & $\mathrm{I} .2 \mathrm{I} \pm 0.0 \mathrm{I}$ & 0.36 \\
\hline Inferior frontal cortex & $1.22 \pm 0.03^{*}$ & $1.17 \pm 0.01$ & 0.06 & $1.22 \pm 0.03$ & $\mathrm{I} .17 \pm 0.0 \mathrm{I}$ & 0.06 \\
\hline Sensorimotor cortex & $1.16 \pm 0.03$ & $1.12 \pm 0.01$ & 0.08 & $1.16 \pm 0.03$ & $\mathrm{I} .12 \pm 0.0 \mathrm{I}$ & 0.16 \\
\hline Broca region & $1.24 \pm 0.04$ & $1.20 \pm 0.01$ & 0.25 & $1.24 \pm 0.03$ & $\mathrm{I} .20 \pm 0.0 \mathrm{I}$ & 0.29 \\
\hline Anterior cingulate cortex & $1.15 \pm 0.03$ & $1.17 \pm 0.01$ & 0.69 & $1.16 \pm 0.03$ & $1.17 \pm 0.01$ & 0.74 \\
\hline Posterior cingulate cortex & $1.36 \pm 0.04$ & $1.34 \pm 0.01$ & 0.67 & $1.35 \pm 0.04$ & $1.34 \pm 0.01$ & 0.86 \\
\hline Superior parietal cortex & $1.06 \pm 0.03$ & $\mathrm{I} .0 \mathrm{I} \pm 0.0 \mathrm{I}$ & 0.06 & $1.04 \pm 0.02$ & $1.02 \pm 0.01$ & 0.23 \\
\hline Inferior parietal cortex & $1.10 \pm 0.03$ & $1.05 \pm 0.01$ & 0.10 & $1.09 \pm 0.03$ & $1.06 \pm 0.01$ & 0.19 \\
\hline Parietal temporal cortex & $1.15 \pm 0.04$ & $1.12 \pm 0.01$ & 0.31 & $1.16 \pm 0.03$ & $\mathrm{I} .12 \pm 0.0 \mathrm{I}$ & 0.20 \\
\hline Superior lateral temporal cortex & $1.21 \pm 0.03$ & $1.17 \pm 0.01$ & 0.19 & $1.2 \mathrm{I} \pm 0.03$ & $1.17 \pm 0.01$ & 0.17 \\
\hline Inferior lateral anterior temporal cortex & $1.00 \pm 0.03$ & $0.97 \pm 0.01$ & 0.27 & $\mathrm{I} .0 \mathrm{I} \pm 0.02$ & $0.97 \pm 0.01$ & 0.12 \\
\hline Inferior lateral posterior temporal cortex & $1.07 \pm 0.03$ & $1.05 \pm 0.01$ & 0.35 & $1.08 \pm 0.02$ & $1.05 \pm 0.01$ & 0.21 \\
\hline Anterior medial temporal cortex & $0.82 \pm 0.02$ & $0.8 I \pm 0.07$ & 0.55 & $0.83 \pm 0.02$ & $0.8 \mathrm{I} \pm 0.0 \mathrm{I}$ & 0.21 \\
\hline Posterior medial temporal cortex & $1.02 \pm 0.03$ & $0.98 \pm 0.01$ & 0.34 & $1.02 \pm 0.02$ & $\mathrm{I} .00 \pm 0.0 \mathrm{I}$ & 0.19 \\
\hline Primary visual cortex & $1.18 \pm 0.06 *$ & $1.27 \pm 0.01$ & 0.04 & $1.19 \pm 0.04 *$ & $1.27 \pm 0.02$ & 0.049 \\
\hline Associated visual cortex & $1.17 \pm 0.04$ & $1.15 \pm 0.01$ & 0.63 & $1.17 \pm 0.03$ & $1.15 \pm 0.01$ & 0.59 \\
\hline
\end{tabular}

Notes: Single-factor analysis by variance and adjusting confounding factors by covariate analysis. $* p<0.05$.

Abbreviations: ${ }^{18}$ F-FDG PET-CT, 18-fludeoxyglucose positron emission tomography-computed tomography; PD, Parkinson's disease; FOG, freezing of gait. 


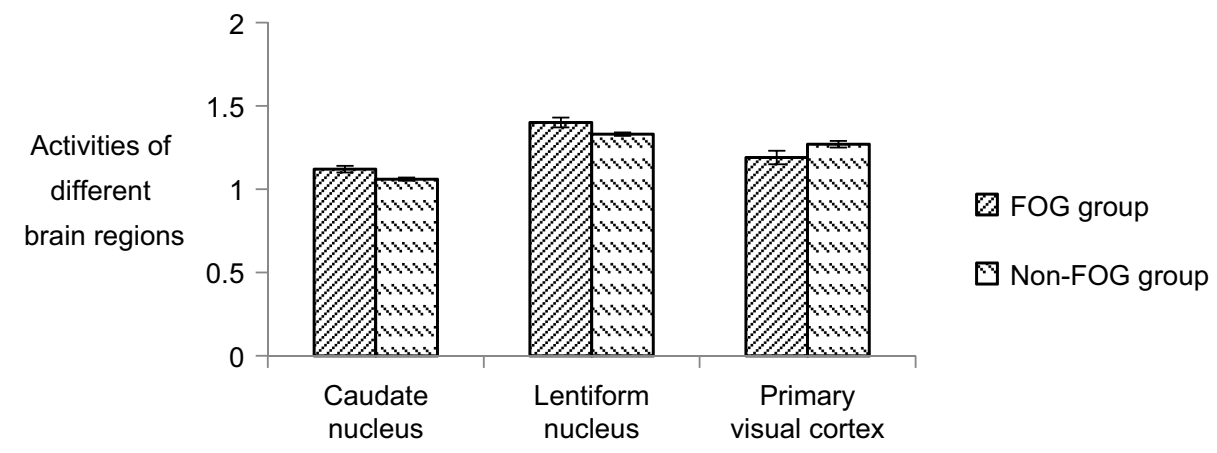

Figure 6 Activities of different brain regions between the two groups. Abbreviation: FOG, freezing of gait.

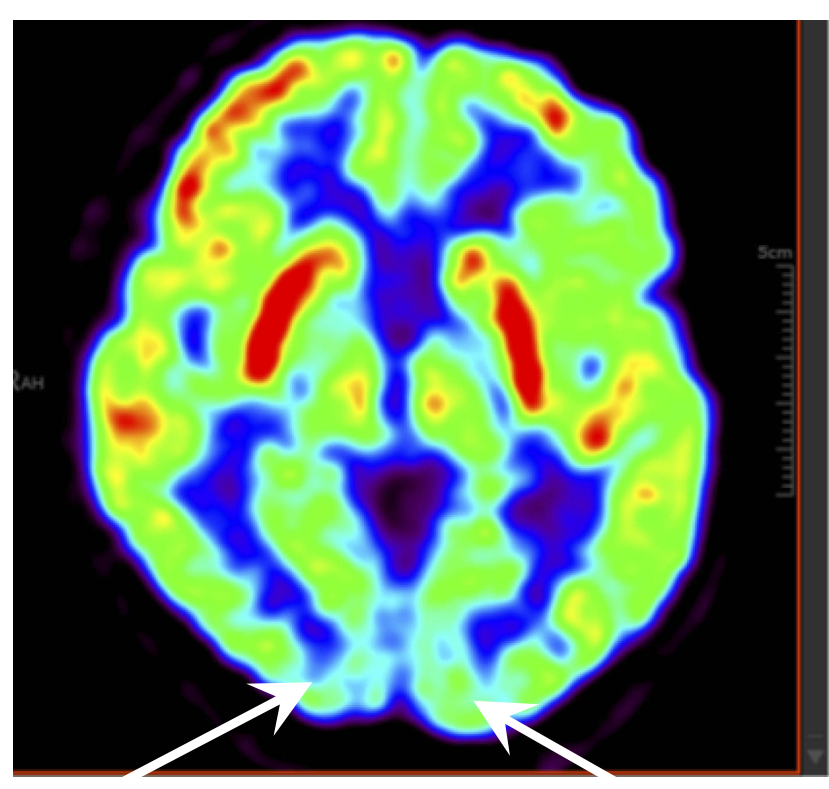

Figure 7 PET-CT image of Hoehn-Yahr stage 3 patient with freezing of gait. Two white arrows show hypometabolism of bilateral primary visualcortex.

Abbreviation: PET-CT, positron emission tomography-computed tomography.

stage and UPDRS-III. The reason for this was that patients with PD could be restored to nearly normal life by reasonable plans with anti-Parkinson drugs, rehabilitation, and psychological support. Therefore, the Hoehn-Yahr and UPDRS-III scores could be reduced by appropriate therapy. This result was contrary to previous studies in which FOG was strongly related to the severity of PD according to Hoehn-Yahr and UPDRS-III scores, and the bias may come from the assessment time and sample selection.

The pathophysiology of FOG is not well understood but is thought to also be driven by impairments of multiple brain areas. Previous studies reported that FOG was related to cognitive dysfunction, for which cognitive training is an effective intervention and may lessen the severity

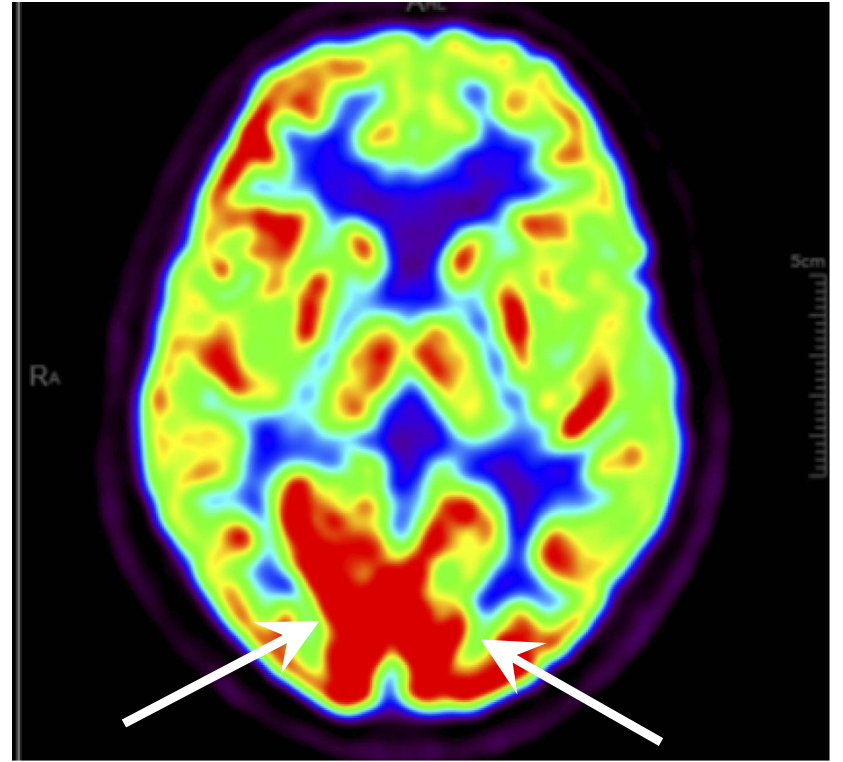

Figure 8 PET-CT imageof Hoehn-Yahr stage 3 patient without freezing of gait. Two white arrows indicate hypermetabolism of bilateral primary visualcortex. Abbreviation: PET-CT, positron emission tomography-computed tomography.

of FOG. ${ }^{5,6,16}$ Then, it is plausible that cognitive impairment may partly underlie the mechanisms of FOG. Our data also found that the scores of the MMSE and MoCA were significantly decreased in patients with FOG. This indicated that cognitive function was involved in the onset of FOG.

PET studies have allowed us to gain important insights into the functions of the dopaminergic system and nondopaminergic system in PD. VMAT2 is the protein responsible for transporting dopamine and is likely to allow us to directly assess degeneration of the substantia nigra neuron and dopamine level in the living PD brain. Up to date, few studies on the VMAT2 distribution or ${ }^{18} \mathrm{~F}$-FDG metabolism by PET-CT imaging have been performed in $\mathrm{PD} .^{23}$ 


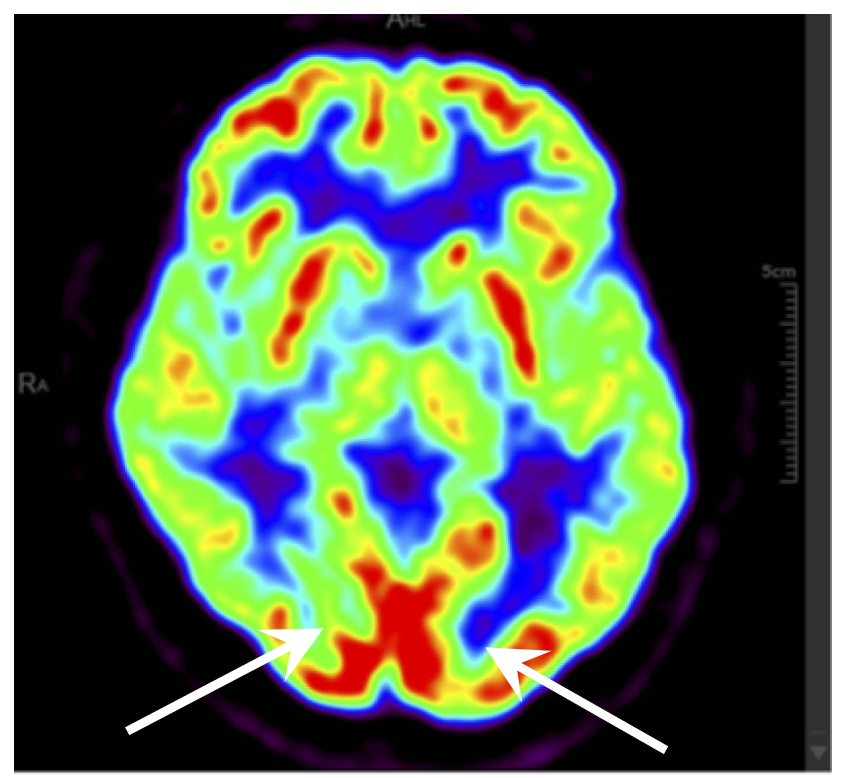

Figure 9 PET-CT image of a normal person. Two white arrows show the normal metabolism of primary visualcortex.

Abbreviation: PET-CT, positron emission tomography-computed tomography

Our data showed that the VMAT2 distribution with ${ }^{18}$ F-AV133 was decreased in PD patients with FOG compared with those without FOG. This indicated that the degeneration of the nigrostriatal system was more severe and the dopamine level was significantly lower in PD patients with FOG compared with those without FOG. The reason for this was that the dopamine level was markedly depleted and motor symptoms were remarkably aggravated as the substantia nigra was largely impaired by Lewy bodies. This was consistent with our clinical observations in which patients with FOG had a longer duration and more doses of dopaminergic drugs.

The reason for selecting the cerebellum as the reference region was that the Lewy body of cerebellar pathology has not been demonstrated unequivocally in different stages of PD. ${ }^{24,25}$ The mean uptake ratios of different brain regions were defined by comparison with the reference region. We found that the uptake ratios of the caudate nucleus and lentiform nucleus were higher in patients with FOG than in those without FOG by ${ }^{18}$ F-FDG PET-CT imaging. Increased metabolism of the caudate nucleus and lentiform nucleus may relate to the plastic changes of these areas in order to compensate for the decreased dopamine level of the nigrostriatal system. $^{26,27}$ Our data also showed that the uptake ratio of the primary visual cortex was significantly lower in patients with FOG compared with those without FOG, and indicated that the impairment of the primary visual cortex partly accounted for the occurrence of FOG. This was consistent with previous reports that FOG came from the result of cognitive or sensorimotor dysfunction, and rehabilitation with visual cues could partly alleviate FOG. ${ }^{28,29}$

Assessment of FOG in the context of PD remains illdefined and poorly understood, making generalizations difficult. Various methods of FOG assessment are implemented across different studies. ${ }^{30}$ Another limitation of our study came from the sample selection, with all participants being inpatients of our PD unit.

In summary, this was the first study to investigate the activities of different brain regions by PET-CT and found that the dopaminergic pathway and primary visual cortex may be involved in the pathogenesis of FOG. FOG was more common in the advanced stage of PD. The ratio of FOG was strongly associated with the duration. A large dose of dopaminergic drugs was needed to alleviate the motor symptoms of PD with FOG. The dopamine level, which was shown by VMAT2 with ${ }^{18}$ F-AV133 PET-CT, was decreased significantly in patients with FOG. This result also implied that FOG was not just a motor symptom but an important predictor for the advanced stage of PD. Metabolism of the primary visual cortex by ${ }^{18}$ F-FDG PET-CT decreased significantly in patients with FOG compared with those without FOG. This provided evidence that the management of FOG needs to not only provide an appropriate dose of dopaminergic drugs, but also take an active part in cognitive training with visual cues.

\section{Acknowledgments}

The project was supported by the National Natural Science Foundation of China (30970926), National R\&D Program of China (2017YFC0840100 and 2017YFC0840105), and the Foundation Clinical Research of Capital Medical University (number: 14JL19).

\section{Author contributions}

All authors made substantial contributions to conception and design, acquisition of data, or analysis and interpretation of data; took part in drafting the article or revising it critically for important intellectual content; gave final approval of the version to be published; and agree to be accountable for all aspects of the work.

\section{Disclosure}

The authors report no conflicts of interest in this work. 


\section{References}

1. Nutt JG, Bloem BR, Giladi N, Hallett M, Horak FB, Nieuwboer A. Freezing of gait: moving forward on a mysterious clinical phenomenon. Lancet Neurol. 2011;10:734-744. doi:10.1016/S14744422(11)70143-0

2. Espay AJ, Fasano A, van Nuenen BF, Payne MM, Snijders AH, Bloem BR. "On" state freezing of gait in Parkinson disease: a paradoxical levodopa-induced complication. Neurology. 2012;78:454-457. doi:10.1212/WNL.0b013e3182477ec0

3. Schaafsma JD, Balash Y, Gurevich T, Bartels AL, Hausdorff JM, Giladi N. Characterization of freezing of gait subtypes and the response of each to levodopa in Parkinson'sdisease. Eur J Neurol. 2003;10:391-398.

4. Ambani LM, Van Woert MH. Start hesitation-a side effect of longterm levodopa therapy. $N$ Engl $J$ Med. 1973;288:1113-1115. doi:10.1056/NEJM197305242882108

5. Gilat M, Lígia Silva de Lima A, Bloem BR, Shine JM, Nonnekes J, Lewis SJG. Freezing of gait: promising avenues for future treatment Parkinsonism Relat Disord. 2018;52:7-16. doi:10.1016/j. parkreldis.2018.03.009

6. Cucca A, Biagioni MC, Fleisher JE, et al. Freezing of gait in Parkinson's disease: from pathophysiology to emerging therapies. Neurodegener Dis Manag. 2016;6:431-446. doi:10.2217/nmt-20160018

7. Nieuwboer A, Giladi N. Characterizing freezing of gait in Parkinson's disease: models of an episodic phenomenon. Mov Disord. 2013;28:1509-1519. doi:10.1002/mds.25683

8. Bartels AL, Leenders KL. Brain imaging in patients with freezing of gait. Mov Disord. 2008;23(Suppl 2):S461-S467. doi:10.1002/ mds. 21912

9. Park HK, Kim JS, Im KC, et al. Functional brain imaging in pure akinesia with gait freezing: $\left[{ }^{18} \mathrm{~F}\right]$ FDG PET and $\left[{ }^{18} \mathrm{~F}\right]$ FP-CIT PET analyses. Mov Disord. 2009;24:237-245. doi:10.1002/mds.22347

10. Pikstra ARA, van der Hoorn A, Leenders KL, de Jong BM. Relation of ${ }^{18}$-F-Dopa PET with hypokinesia-rigidity, tremor and freezing in Parkinson's disease. Neuroimage Clin. 2016;11:68-72. doi:10.1016/j. nicl.2016.01.010

11. Ouchi Y, Kanno T, Okada H, et al. Changes in dopamine availability in the nigrostriatal and mesocortical dopaminergic systems by gait in Parkinson's disease. Brain. 2001;124:784-792.

12. Kilbourn MR, Sherman PS, Kuszpit K. In vivo measures of dopaminergic radioligands in the rat brain: equilibrium infusion studies. Synapse. 2002;43:188-194. doi:10.1002/syn.10039

13. Kilbourn MR. Long-term reproducibility of in vivo measures of specific binding of radioligands in rat brain. Nucl Med Biol. 2004;31:591-595. doi:10.1016/j.nucmedbio.2004.02.003

14. Perez-Lloret S, Negre-Pages L, Damier P, et al. Prevalence, determinants, and effect on quality of life of freezing of gait in Parkinson disease. JAMA Neurol. 2014;71:884-890. doi:10.1001/ jamaneurol.2014.753

15. Okuma Y. Freezing of gait and falls in Parkinson's disease. J Parkinsons Dis. 2014;4:255-260. doi:10.3233/JPD-130282

16. Giladi N, McDermott MP, Fahn S, et al. Freezing of gait in PD: prospective assessment in the DATATOP cohort. Neurology. 2001;56:1712-1722.

17. Macht M, Kaussner Y, Möller JC, et al. Predictors of freezing in Parkinson's disease: a survey of 6,620 patients. Mov Disord. 2007;22:953-956. doi:10.1002/mds.21458
18. Fasano A, Herman T, Tessitore A, Strafella AP, Bohnen NI. Neuroimaging of Freezing of Gait. $J$ Parkinsons Dis. 2015;5:241-254. doi:10.3233/JPD-150536

19. Forsaa EB, Larsen JP, Wentzel-Larsen T, Alves G. A 12-year population-based study of freezing of gait in Parkinson's disease. Parkinsonism Relat Disord. 2015;21:254-258. doi:10.1016/j. parkreldis.2014.12.020

20. Amboni M, Stocchi F, Abbruzzese G, et al. Attar features of self-reported freezing of gait in Parkinson disease: the DEEP FOG study. Parkinsonism Relat Disord. 2015;21:644-649. doi:10.1016/j. parkreldis.2015.03.028

21. Ou R, Wei Q, Cao B, et al. Predictors of freezing of gait in Chinese patients with Parkinson's disease. Brain Behav. 2018;8(3):e00931. doi:10.1002/brb3.931

22. Sun Q, Wang T, Jiang TF, et al. Clinical profile of Chinese long-term parkinson's disease survivors with 10 years of disease duration and Beyond. Aging Dis. 2018;9:8-16. doi:10.14336/AD.2017.0204

23. Brooks DJ. Monitoring neuroprotection and restorative therapies in Parkinson's disease with PET. J Neural Transm Suppl. 2000; (60):125-137.

24. Dickson DW. Parkinson's disease and parkinsonism: neuropathology. Cold Spring Harb Perspect Med. 2012;2(8):pii: a009258. doi:10.1101/cshperspect.a009258

25. Braak H, Del Tredici K. Neuropathological staging of brain pathology in sporadic parkinson's disease: separating the wheat from the chaff. J Parkinsons Dis. 2017;7(s1):S71-S85. doi:10.3233/JPD179001

26. Villalba RM, Smith Y. Loss and remodeling of striatal dendritic spines in Parkinson's disease: from homeostasis to maladaptive plasticity? J Neural Transm (Vienna). 2018;125:431-447. doi:10.1007/ s00702-017-1735-6

27. Beck G, Singh A, Papa SM. Dysregulation of striatal projection neurons in Parkinson's disease. $J$ Neural Transm (Vienna). 2018;125:449-460. doi:10.1007/s00702-017-1744-5

28. Barthel C, Nonnekes J, van Helvert M, et al. The laser shoes: A new ambulatory device to alleviate freezing of gait in Parkinson disease. Neurology. 2018;90:e164-e171. doi:10.1212/WNL.0000000000004 795

29. Ginis P, Nackaerts E, Nieuwboer A, Heremans E. Cueing for people with Parkinson's disease with freezing of gait: A narrative review of the state-of-the-art and novel perspectives. Ann Phys Rehabil Med. 2018;61:407-413. doi:10.1016/j.rehab.2017.08.002

30. Dahodwala N, Siderowf A, Baumgarten M, Abrams A, Karlawish J. Screening questionnaires for parkinsonism: a systematic review. Parkinsonism Relat Disord. 2012;18:216-224. doi:10.1016/j. parkreldis.2011.09.003

31. Gao R, Zhang G, Chen X, et al. CSF biomarkers and its associations with 18F-AV133 cerebral VMAT2 binding in parkinson's disease-a preliminary report. PLoS One. 2016;11:e0164762. doi:10.1371/journal.pone. 0164762

32. Alexander PK, Lie Y, Jones G, et al. Management impact of imaging brain vesicular monoamine transporter type 2 in clinically uncertain parkinsonian syndrome with $18 \mathrm{~F}-\mathrm{AV} 133$ and PET. J Nucl Med. 2017;58:1815-1820. doi:10.2967/jnumed.116.189019

33. Xu SS, Alexander PK, Lie Y, et al. Diagnostic accuracy of imaging brain vesicular monoamine transporter type 2 (VMAT2) in clinically uncertain parkinsonian syndrome (CUPS): a 3-year follow-up study in community patients. BMJ Open. 2018;8:e25533. doi:10.1136/ bmjopen-2018-025533 


\section{Publish your work in this journal}

Neuropsychiatric Disease and Treatment is an international, peerreviewed journal of clinical therapeutics and pharmacology focusing on concise rapid reporting of clinical or pre-clinical studies on a range of neuropsychiatric and neurological disorders. This journal is indexed on PubMed Central, the 'PsycINFO' database and CAS, and is the official journal of The International Neuropsychiatric Association (INA). The manuscript management system is completely online and includes a very quick and fair peer-review system, which is all easy to use. Visit http://www.dovepress.com/testimonials.php to read real quotes from published authors. 